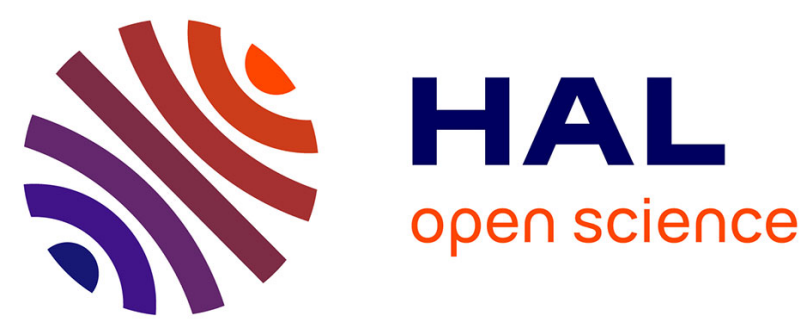

\title{
Traitement du syndrome des jambes sans repos nouvellement diagnostiqué
}

\author{
N. Limousin, M. Flamand, C. Schröder, C. Charley Monaca
}

\section{To cite this version:}

N. Limousin, M. Flamand, C. Schröder, C. Charley Monaca. Traitement du syndrome des jambes sans repos nouvellement diagnostiqué. Médecine du sommeil, 2019, 16, pp.106 - 113. 10.1016/j.msom.2018.10.003 . hal-03486367

\section{HAL Id: hal-03486367 https://hal.science/hal-03486367}

Submitted on 20 Dec 2021

HAL is a multi-disciplinary open access archive for the deposit and dissemination of scientific research documents, whether they are published or not. The documents may come from teaching and research institutions in France or abroad, or from public or private research centers.
L'archive ouverte pluridisciplinaire HAL, est destinée au dépôt et à la diffusion de documents scientifiques de niveau recherche, publiés ou non, émanant des établissements d'enseignement et de recherche français ou étrangers, des laboratoires publics ou privés.

\section{다)(1) $(5$}

Distributed under a Creative Commons Attribution - NonCommerciall 4.0 International 
Version of Record: https://www.sciencedirect.com/science/article/pii/S1769449318302449

Manuscript_1a64e4c9d2a4e854ac368c933072ee3a

Dochead : Consensus français sous l'égide de la SFRMS sur le Syndrome des Jambes sans Repos ou maladie de Willis Ekbom

Titre

Traitement du syndrome des jambes sans repos nouvellement diagnostiqué*

Treatment of newly diagnosed restless legs syndrome

Auteurs: Nadège LIMOUSIN ${ }^{1}$, Mathilde FLAMAND², Carmen SCHRÖDER ${ }^{3}$, Christelle CHARLEY MONACA $^{2 *}$

*Correspondance : Christelle CHARLEY MONACA ; christelle.monaca@chru-lille.fr

\section{Affiliations :}

${ }^{1}$ Neurophysiologie Clinique, CHU de Tours, e-mail : n.limousin-champfailly@chu-tours.fr

${ }^{2}$ Neurophysiologie Clinique, CHU, Université de Lille, INSERM UMR 1171, Lille, France - e-mails : mathilde.flamand@chru-lille.fr ; christelle.monaca@chru-lille.fr

${ }^{3}$ Service de Psychiatrie de l'Enfant et de l'Adolescent, Pôle Psychiatrie, Santé Mentale et Addictologie , Hôpitaux Universitaires de Strasbourg et Université de Strasbourg , 1, Place de l'Hôpital 67091 Strasbourg Cedex ; CNRS UPR 3212, Equipe 9, Institut des Neurosciences Cellulaires et Intégratives (INCI), 5, rue Blaise Pascal, 67084 Strasbourg Cedex - e-mail : schroderc@unistra.fr

\section{Déclaration des liens d'intérêt :}

Nadège LIMOUSIN : aucun

Mathilde FLAMAND : aucun

Carmen SCHRÖDER : Coordonnatrice et investigatrice d'études (Neurim), oratrice rémunérée (Janssen, Biocodex)

Christelle CHARLEY MONACA : experte et oratrice pour UCB Pharma, experte pour Philips et ResMed

Mots-clés: syndrome des jambes sans repos, agonistes dopaminergiques, ligands a2ঠ, opiacés 
Keywords: Restless legs syndrome, Dopaminergic agonists, a2ס ligands, Opioids

\section{Abréviations :}

$A D$ : agonistes dopaminergiques

AMM : autorisation de mise sur le marché

IRLS : International Restless Legs Syndrome Study Group rating scale

IRLSSG : International Restless Legs Syndrome Study Group

MPM : mouvements périodiques des membres

MPS : mouvements périodiques au cours du sommeil

RLS QoL : Restless Legs Syndrome Quality of Life

SF-36 : Short Form - 36

SJSR : syndrome des jambes sans repos

${ }^{*}$ Ce consensus a déjà fait l'objet d'une publication en anglais dans Revue Neurologie. Pour référencer ce texte, merci de citer l'article princeps LIMOUSIN N., FLAMAND M., SCHRÖDER C., CHARLEY MONACA C., French consensus: Treatment of newly diagnosed restless legs syndrome, Rev Neurol, $2018 ; 174$ : 515-521. http://doi.org/10.2016/j.neurol.2018.06.002 


\section{Résumé}

La prise en charge thérapeutique du syndrome des jambes sans repos (SJSR) ne doit être envisagée qu'après un diagnostic de certitude. Le SJSR doit donc être phénotypé précisément, une carence martiale doit être recherchée systématiquement et les facteurs favorisants doivent être éliminés lorsque cela est possible.

Un traitement médicamenteux sera envisagé pour les formes sévères ou très sévères. Le traitement médicamenteux sera basé sur l'utilisation d'agonistes dopaminergiques, et/ou de ligands $\alpha 2 \delta$-1, et/ou d'opiacés. En première intention, le traitement sera une monothérapie, à faible posologie et le choix de la molécule sera fonction de l'interrogatoire et du bilan réalisé.

\section{Abstract}

Treatment of restless legs syndrome (RLS) must only be considered after a definite positive diagnosis. The RLS phenotype must be characterised precisely, iron deficiency always tested for, and aggravating factors eliminated when possible.

Medical treatment is considered for severe or very severe forms and based on dopaminergic agonists, $\alpha 2 \delta-1$ ligands and / or opioids. First line treatment will be a low-dose monotherapy and the choice of treatment depends on the results of the clinical examination and investigations. 


\section{Article}

\section{Introduction}

Avant d'envisager la mise en place d'un traitement médicamenteux, il est nécessaire de :

- poser un diagnostic positif de certitude de syndrome des jambes sans repos (SJSR) ;

- expliquer au patient sa maladie et la physiopathologie de cette dernière ;

- rechercher les facteurs aggravants du SJSR ;

- $\quad$ évaluer la sévérité du SJSR. II est important de souligner que la majorité des études ont évalué l'efficacité des traitements dans les formes modérées à très sévères (IRLS > 10) selon le score obtenu à l'échelle de sévérité internationale du SJSR (IRLSSG). En pratique, il faut prendre en compte les répercussions sur la qualité de vie des patients (sommeil, vie sociale, humeur) pour décider de la prise en charge. Ces aspects sont certes rapportés dans l'échelle de sévérité mais ils doivent être systématiquement rediscutés avec le patient avant de prendre une décision thérapeutique.

Ces 3 étapes sont détaillées dans l'article spécifique sur le diagnostic de SJSR.

II faut également :

- arrêter, si cela est possible, les traitements aggravants : les antidépresseurs (toutes classes), les neuroleptiques, le lithium, les anti-histaminiques, l'oxybate de sodium.

- donner au patient des conseils d'hygiène de vie et ce, quelque soit la sévérité du SJSR (Tableau 1). Elles permettront de minimiser la symptomatologie du SJSR. Ces conseils sont basés sur la littérature mais aussi sur l'expérience de notre groupe d'experts.

\section{Prise en charge d'une éventuelle carence martiale}

Toujours avant d'initier un traitement médicamenteux, il est nécessaire de réaliser un bilan martial. La plupart des études ont évalué l'effet de la supplémentation martiale pour une ferritinémie $<50 \mu \mathrm{g} / \mathrm{mL}$. En pratique, certains patients tirent bénéfice d'une supplémentation martiale avec une ferritinémie $<75 \mu \mathrm{g} / \mathrm{mL}$. L'efficacité d'une supplémentation martiale dans le SJSR est connue depuis longtemps. II convient également de rechercher la cause de la carence martiale et la traiter. En première intention, nous préconisons la supplémentation orale (TARDYFERON ${ }^{\circledR}$, TARDYFERON B9 $^{\circledR}$ ) 
avec un contrôle de la ferritine à 3 mois. En cas d'inefficacité d'une supplémentation martiale per os, la voie intraveineuse peut être considérée comme une alternative. La supplémentation se fait selon les habitudes de chacun ou selon les produits disponibles dans le centre hospitalier.

Elle est proposée en milieu hospitalier en raison du risque anaphylactique encouru par le patient, risque toutefois moins fréquent avec la formulation à base de carboxymaltose ferrique. Cette formulation est la seule à avoir montré une efficacité supérieure au placebo dans les études randomisées en double aveugle [1,2], (perfusions de fer à une dose maximale de $1000 \mathrm{mg}$ en une seule fois). On contrôlera la ferritinémie 4 à 6 semaines après la perfusion.

\section{Cas particuliers}

Si les patients ont une forme très grave de SJSR associé à une hypoferritinémie, une supplémentation intraveineuse peut être proposée d'emblée.

Selon le terrain (par exemple sujet âgé), si la ferritinémie est très basse un hémoccult pourrait être réalisé à la recherche d'une cause digestive à la carence martiale.

Pour les enfants, la supplémentation orale est à privilégier. II est important de privilégier la bonne tolérance du traitement, et notamment l'apparition de douleurs digestives ou crampes et en vérifiant le transit de l'enfant. Pour l'enfant, on peut débuter généralement avec du FERROSTRANE ${ }^{\circledR}$ à 1-3 cuillères à café par jour (34-102 mg/j) car il serait celui qui entraîne le moins d'effet sur le transit digestif et car il peut facilement être modulé. Si ce traitement provoque des troubles du transit, on peut proposer la prise de comprimés enrobés FUMAFER ${ }^{\circledR} 66 \mathrm{mg}$ ou, pour ceux qui n'arrivent pas à avaler, du FUMAFER ${ }^{\circledR}$ en poudre (1-3 cuillères-dose/j, soit 33-99 mg/j) ou encore du TIMOFEROL ${ }^{\circledR} 50 \mathrm{mg}$ en gélule (car la gélule peut être ouverte). En cas d'intolérance digestive, une perfusion intra-veineuse $\left(V_{E N O F E R r}{ }^{\circledR}\right)$ peut être considérée en deuxième intention.

Pour les patients ayant une forme sévère ou très sévère de SJSR sans carence martiale ou avec une supplémentation ferrique insuffisamment efficace, un traitement médicamenteux est à envisager. Trois classes de médicaments sont disponibles : les agents dopaminergiques, certains anti-épileptiques et les opioïdes.

\section{Les agents dopaminergiques}


Les agents dopaminergiques comportent la lévodopa et les agonistes dopaminergiques. Seules 3 molécules ont l'autorisation de mise sur le marché (AMM) en France : l'ADARTREL ${ }^{\circledR}$, le SIFROL ${ }^{\circledR}$ et le NEUPRO ${ }^{\circledast}$. Aucun n'est à ce jour remboursé dans l'indication du syndrome des jambes sans repos.

\subsection{La lévodopa}

Plusieurs études randomisées contre placébo ont prouvé l'efficacité de la lévodopa pour traiter le SJSR. Du fait du risque d'automédication abusive et du risque de syndrome d'augmentation, nous ne recommandons pas l'utilisation de ce traitement dans le syndrome des jambes sans repos.

\subsection{Les agonistes dopaminergiques}

Les agonistes dopaminergiques peuvent être classés en deux groupes : dérivés de l'ergot de seigle et les non ergot de seigle.

Les dérivés de l'ergot de seigle ne sont pas recommandés dans le traitement du SJSR du fait des effets secondaires : risque de fibrose (hépatique, pulmonaire, péricardique et rétropéritonéal) et de valvulopathie cardiaque.

Les agonistes dopaminergiques non ergotés disponibles en France et ayant l'AMM dans l'indication du SJSR sont l'ADARTREL ${ }^{\circledR}$, le SIFROL ${ }^{\circledR}$ et le NEUPRO ${ }^{\circledR}$. La quantité et la qualité des articles scientifiques ont conduit à l'utilisation de ces traitements dans les formes modérées à très sévères en première intention dans le SJSR depuis plus de 10 ans.

\subsubsection{Le pramipexole (sous la dénomination SIFROL®)}

Le pramipexole est un agoniste D1, D2 et D3 des récepteurs dopaminergiques, avec une affinité plus spécifique sur les récepteurs D3. Sa demi-vie est de 8 à 12 heures et son délai d'action entre 1 à 2 heures après la prise. Huit études avec un niveau de preuve $1 \mathrm{~A}$ ont montré son efficacité à court terme et moyen terme ( 6 mois) dans l'indication du SJSR. Des doses variant de 0,5-0,75 mg ont montré une amélioration de la latence d'endormissement, mais pas d'effet sur l'efficacité du sommeil ni le temps de sommeil total [3]. Le pramipexole améliore les scores de qualité de vie (échelles Restless Legs Syndrome Quality of Life - RLS QoL - et SF-36) [3-7] mais n'améliore pas celui de l'échelle de dépression de Beck ni l'échelle d'anxiété HAD (Hospital Anxiety and Depression scale). Dans une étude sur 6 mois chez 331 patients avec un SJSR idiopathique, le score de sévérité reste significativement amélioré [8]. 
II dispose d'une AMM en France pour le traitement symptomatique du SJSR idiopathique modéré à sévère (score IRLS > 10), mais il n'est pas remboursé à ce jour dans l'indication du SJSR. Pour le traitement du SJSR, seuls les comprimés dosés à 0,18 mg sont utilisés. La dose initiale recommandée est de $0,09 \mathrm{mg}(1 / 2$ comprimé de SIFROL $\circledast$ dosé à $0,18 \mathrm{mg})$, à prendre une fois par jour 1 à 2 heures avant l'apparition des symptômes. Si cette dose s'avère insuffisante, elle peut être augmentée tous les 4 à 7 jours, jusqu'à la dose maximale de 0,54 mg par jour.

Dans les formes où les symptômes s'étendent sur plus de 8 heures, du SIFROL ${ }^{\circledR}$ à libération prolongée peut être proposé (mais sans AMM).

\subsubsection{Le ropinirole (sous la dénomination ADARTREL ${ }^{\circledR}, \operatorname{REQUIP}^{\circledR}$ )}

Le ropinirole est un agoniste dopaminergique qui agit principalement sur les récepteurs D2 et D3 avec une affinité plus importante pour les récepteurs D3. II atteint sa concentration plasmatique maximum entre 1 à 2 heures après la prise orale et a une demi-vie de 6 heures. Dans les études, une dose moyenne de ropinirole entre 2 et $3 \mathrm{mg} /$ jour améliore les symptômes du SJSR (évalués sur l'échelle IRLS, sur l'impression clinique globale, sur l'impression globale du patient) et diminue l'index des mouvements périodiques des jambes. Le ropinirole est recommandé dans le traitement du SJSR selon les consensus américain et européen avec le même degré de recommandation que le pramipexole $[9,10]$.

Seul le ropinirole dans sa dénomination $\operatorname{ADARTREL}^{\circledR}$ a l'AMM pour le traitement du SJSR modéré à sévère, mais il n'est plus remboursé à ce jour. II se présente sous forme de comprimés dosés à $0,25 \mathrm{mg}, 0,50 \mathrm{mg}, 1 \mathrm{mg}$ et $2 \mathrm{mg}$. La dose initiale recommandée est de $0,25 \mathrm{mg}$ (1 comprimé dosé à $0,25 \mathrm{mg}$ ), une fois par jour pendant deux jours. Si cette dose est bien tolérée, elle sera augmentée à $0,5 \mathrm{mg} / \mathrm{jour}$ jusqu'à la fin de la première semaine, puis si nécessaire à $1 \mathrm{mg} / \mathrm{jour}$ la deuxième semaine. La dose peut ensuite être augmentée de 0,5 mg par semaine sur les deux semaines suivantes, jusqu'à atteindre une dose de $2 \mathrm{mg} / \mathrm{jour}$.

Le REQUIP ${ }^{\circledR}$ LP 2 mg peut avoir un intérêt pour le traitement des SJSR dont les symptômes durent plus de 8 heures par 24 heures mais cette indication n'a pas d'AMM.

\subsubsection{La rotigotine (sous la dénomination NEUPRO ${ }^{\circledR}$ )}

II s'agit d'un agoniste D1, D2, D3, D4 et D5 plus $5 \mathrm{HT}_{1 \mathrm{~A}}$ et $\alpha-2$ adrénergique. Le dispositif est transcutané, à changer quotidiennement. Ce traitement a été étudié dans 5 études randomisées 
contre placebo d'une durée d'1 semaine à 6 mois. Dans une étude sur 6 semaines, la rotigotine dosée à 1, 2, 3, 4 mg/24h améliorait le score de sévérité du syndrome des jambes sans repos IRLS (-15,3 \pm $10,-15,7 \pm 9,5,17,3 \pm 10,5,14,9 \pm 10,3$ respectivement) et était significativement plus efficace que le placebo. Une amélioration de la qualité de vie (mesurée par le score RLS QoL) était également significativement retrouvée [11]. Une étude européenne sur 6 mois retrouvait une efficacité de 1 à $3 \mathrm{mg}$ de rotigotine avec une amélioration significative du score IRLS et de l'impression clinique globale, item 1 ; l'amélioration du score de l'échelle de qualité de vie était dose dépendante avec une amélioration moyenne du score RLS QoL par rapport au début de 15,43 $\pm 14,04$ contre 7,3 $\pm 13,5$ sous placebo [12]. Une étude avec contrôle polysomnographique retrouvait une diminution significative de l'index de mouvements périodiques des membres inférieurs éveillants sous rotigotine [13].

II n'y a pas d'étude à moyen et long terme de classe I, cependant il existe une phase d'extension en ouvert d'une étude contre placebo, avec une analyse sur 1 an et sur 5 ans [13-15]. A 1 an, la dose moyenne de rotigotine était de $2,8 \pm 1,2 \mathrm{mg} / 24 \mathrm{~h}, 74,6 \%$ des patients continuaient leur traitement à la fin de l'étude. A 5 ans, la dose moyenne de rotigotine était de 3,09 mg/24h. 39\% des patients sous rotigotine n'avaient plus de symptômes à la fin de l'étude, 30\% avaient arrêté le traitement du fait d'effets secondaires et $11 \%$ en raison d'un manque d'efficacité [15]. L'effet secondaire le plus important était une réaction allergique au niveau du site d'application.

Les patchs disponibles en France sont dosés à $2 \mathrm{mg}, 4 \mathrm{mg}, 6 \mathrm{mg}$ et $8 \mathrm{mg}$. La rotigotine a l'AMM pour le SJSR modéré à sévère, mais elle n'est pas remboursée à ce jour. Seuls les patchs de 2 mg (très rarement $4 \mathrm{mg}$ ) sont utilisés dans l'indication du SJSR. Bien que le laboratoire ne recommande pas de couper le patch, dans notre expérience le patch peut être coupé pour obtenir une dose de $1 \mathrm{mg}$ et adapter la posologie. La dose initiale recommandée est d'1 patch de $2 \mathrm{mg}$ (voire d'1/2 patch de $2 \mathrm{mg}$ ) à augmenter si nécessaire à $3 \mathrm{mg}$ (un patch et demi) après un mois. Ce traitement doit être proposé dans les formes où les symptômes s'étendent sur plus de 8 heures. La dose maximale recommandée est de $3 \mathrm{mg}$. Dans certains cas particuliers, des doses de $4 \mathrm{mg}$ se sont montrées nécessaires.

\subsubsection{Les effets secondaires des agonistes dopaminergiques non ergotés}

Ces traitements sont en général mieux tolérés dans le SJSR, possiblement du fait de posologies plus basses et d'une prévalence des comorbidités plus faible que dans la maladie de Parkinson. Les effets 
secondaires sont rares et d'intensité faible à modérée et spécifiques aux agonistes dopaminergiques non ergot de seigle : syndrome de dysrégulation dopaminergique (troubles du contrôle des impulsions), nausées, céphalées, somnolence, hypotension orthostatique, confusion, agitation, hallucinations et nasopharyngite [16-20]. Ces effets sont rapportés chez $23 \%$ des patients et sont habituellement réversibles à l'arrêt ou à la diminution des doses. Les patients doivent systématiquement être avertis du risque de troubles du contrôle des impulsions. Le syndrome d'augmentation est abordé dans un article spécifique et constitue le principal effet secondaire de ces traitements. Ce syndrome d'augmentation peut être prévenu par l'utilisation de faibles posologies d'agonistes dopaminergiques (Tableau 2)

\section{Les anti-épileptiques}

Bien que plusieurs études aient montré l'efficacité des ligands $\alpha 2 \delta-1$, aucun de ces médicaments n'a d'autorisation de mise sur le marché en France pour cette indication.

\subsection{La gabapentine (NEURONTIN ${ }^{\circledR}$ )}

La gabapentine est un agoniste $\alpha 2 \delta-1$, sous-unité d'un canal calcique voltage-dépendant présent sur les neurones. Deux études à court terme d'un niveau de preuve III et IV contre placebo ont montré son efficacité dans le traitement du SJSR $[21,22]$.

La gabapentine a l'AMM dans le traitement de l'épilepsie et des douleurs neuropathiques, mais elle ne l'a pas pour l'indication syndrome des jambes sans repos. Elle se présente en gélule dosée à $100 \mathrm{mg}$, $300 \mathrm{mg}, 400 \mathrm{mg}, 600 \mathrm{mg}$ et $800 \mathrm{mg}$. Il est également disponible sous forme de comprimé dosé à $600 \mathrm{mg}$ et $800 \mathrm{mg}$. Une solution buvable dosée à $250 \mathrm{mg} / 5 \mathrm{ml}$ est également possible. Le traitement doit être initié progressivement jusqu'à une dose unique le soir de 1200 mg maximum ou de 2400 mg par jour en plusieurs prises. Des prises le matin sont à proposer si le patient a des symptômes la journée et si le traitement est bien toléré. Les effets secondaires les plus importants sont somnolence, vertige, fatigue, céphalées et tremblements.

La gabapentine enacarbil est un précurseur de la gabapentine à libération prolongée. Plusieurs études contre placebo de grade I ont montré son efficacité dans le traitement du SJSR [23, 24]. Le groupe international du syndrome des jambes sans repos (International Restless Legs Syndrome Study Group), la Fondation du syndrome des jambes sans repos et le Groupe européen du syndrome 
des jambes sans repos recommandent son utilisation en première ligne car les ligands $\alpha 2 \delta$ n'induisent pas de syndrome d'augmentation contrairement aux agonistes dopaminergiques [25]. Ce traitement n'est pas disponible en France.

\subsection{La prégabaline $\left(\right.$ LYRICA $\left.^{\circledR}\right)$}

La prégabaline est un agoniste $\alpha 2 \delta$-1, sous-unité d'un complexe calcique voltage-dépendant sur les neurones. Trois études de phase I ont montré une efficacité de la prégabaline à court terme (4 à 12 semaines) dans le traitement du SJSR mais le traitement n'a pas été évalué à long terme [26-28]. La prégabaline a l'AMM dans le traitement de l'épilepsie, des douleurs neuropathiques, du trouble anxieux généralisé, mais ne l'a pas dans l'indication du SJSR. Elle se présente sous forme de gélules dosées à 25, 50, 75, 100, 150 mg, 200 mg. Elle est également disponible en solution buvable dosée à $20 \mathrm{mg} / \mathrm{ml}$. La posologie doit être augmentée progressivement en démarrant à $25 \mathrm{mg}$ jusqu'à une posologie maximale de $200 \mathrm{mg} / \mathrm{j}$.

Bien que les études concernant l'efficacité de la prégabaline dans le SJSR soient plus robustes qu'avec la gabapentine, l'expérience des experts rapporte un effet moins sédatif de la gabapentine.

\subsection{Le clonazépam}

Du fait des risques iatrogéniques de ce médicament (pharmacodépendance psychique et physique, altération de certaines fonctions cognitives (vigilance, mémoire, concentration), risque de chute (particulièrement chez le sujet âgé), il n'est pas recommandé en première intention dans le traitement du SJSR. II peut être utilisé en prescription ponctuelle (période d'exacerbation du SJSR par exemple).

\section{Les opioïdes}

Pendant de nombreuses années, les opioïdes ont été utilisés empiriquement comme un traitement alternatif du traitement du SJSR. Malgré une efficacité reconnue depuis longtemps, peu d'études ont évalué l'efficacité des opioïdes sur les symptômes du SJSR. Une seule étude de classe I a évalué l'efficacité de l'oxycodone en association à son antagoniste partiel la naloxone chez 276 patients avec un SJSR douloureux [29]. Les résultats montraient une diminution significative de l'IRLS (score < 15) en comparaison au placebo à 12 semaines, avec le maintien de cette efficacité à 40 semaines. Les 
principaux effets secondaires étaient une fatigue, une constipation, des nausées, des céphalées, une hypersudation, une somnolence, une xérostomie et un prurit, rapportés pendant la phase en double aveugle, avec une diminution de leur fréquence à 40 semaines. Cinq patients ont présenté des effets secondaires graves durant la phase initiale, et 3 durant la phase d'extension. Toutefois, l'association oxycodone/naloxone n'est pas commercialisée en France. L'oxycodone seule a été évaluée dans une étude en double aveugle sur un effectif restreint de 11 patients, avec une efficacité significative par rapport au placebo mesurée par une échelle de sévérité du SJSR subjective non validée [30].

Les opioïdes de niveau I, essentiellement le tramadol (sous la dénomination TOPALGIC ${ }^{\circledR}$ ) peuvent être utilisés en première intention. Le tramadol est commercialisé en gélule de $50 \mathrm{mg}$. Les formes à libération prolongée se présentent sous forme de comprimé dosé à 100 mg, 150 mg, $200 \mathrm{mg}$. Il est également disponible en solution buvable dosée à $100 \mathrm{mg} / \mathrm{ml}$. Les effets secondaires les plus fréquemment rapportés sont : constipation, sédation, développement ou aggravation de troubles respiratoires et risque potentiel d'addiction chez des patients prédisposés.

\section{Quel traitement choisir ? (Figure 1)}

Avant de démarrer un traitement, la physiopathologie mal connue de la maladie doit être clairement expliquée au patient lors de la première entrevue afin qu'il comprenne que sa pathologie ne se guérit pas et que les différents traitements proposés ne sont qu'à visée symptomatique. Le SJSR étant une maladie chronique, sa prise en charge s'inscrit dans cette perspective : le choix du traitement de première intention est guidé à la fois par la condition clinique du patient et par une connaissance des effets adverses, aussi bien à court qu'à long terme du traitement médicamenteux.

Pour les formes légères, nous recommandons l'application des règles d'hygiène de vie, la supplémentation en fer et si nécessaire, l'utilisation de traitement à la demande (tramadol, codéine). Chez les patients avec un SJSR modéré à très sévère, considérer actuellement les agonistes dopaminergiques $(\mathrm{DA})$ comme traitement de première intention ne s'impose plus comme une règle immuable [25], bien que ce soit les seuls traitements qui aient l'AMM dans cette indication en France. Les DA possèdent en effet une efficacité remarquable et reconnue à la fois sur les symptômes du SJSR et les mouvements périodiques au cours du sommeil (MPS) des membres inférieurs souvent associés au SJSR. Cependant, leur marge thérapeutique peut être limitée par un phénomène de réponse paradoxale au-delà d'une certaine posologie. Les DA peuvent également être source d'effets 
secondaires gênants, mais réversibles à l'arrêt du traitement, tels que le trouble du contrôle des impulsions. Ils peuvent enfin être à l'origine du syndrome d'augmentation qui peut nécessiter leur arrêt. Ils peuvent être réservés aux formes très sévères de SJSR, aux patients ayant une dépression comorbide, aux patients insuffisants rénaux, aux patients ayant un SJSR accompagné de MPS à la polysomnographie. II est absolument indispensable de limiter leur posologie (Tableau 2), d'éviter les prises multiples journalières.

Toujours pour le SJSR sévère ou très sévère, certains anti-épileptiques tels que les ligands $\alpha 2 \delta$ peuvent avantageusement remplacer les DA ou leur être associés. Leur efficacité sur les symptômes du SJSR est reconnue [31], mais pas sur les MPS [28]. Du fait de leurs propriétés antalgique, sédative et anxiolytique, les ligands a2ठ peuvent même être proposés en première intention chez les patients avec un SJSR à composante douloureuse, un SJSR associé à une insomnie [25], chez les patients ayant un trouble anxieux généralisé ou des antécédents de troubles du contrôle des impulsions. De plus, les ligands $\alpha 2 \delta$ ont, à notre avis, un meilleur profil de tolérance sur le long cours que les DA. Finalement, les opioïdes peuvent aussi être utilisés en tant qu'alternative thérapeutique. Les opioïdes de palier 2 peuvent être utilisés soit de façon ponctuelle, soit au long cours, soit encore en association avec d'autres familles thérapeutiques. Les opioïdes de palier 3 sont réservés aux formes très sévères de SJSR et à celles résistantes aux autres classes médicamenteuses.

II faut expliquer aux patients que l'objectif thérapeutique est de réduire la plainte de SJSR mais de ne pas éradiquer les symptômes ce qui ne permettrait pas une bonne prise en charge à long terme. Quel que soit le traitement, il doit être prescrit une à deux heures avant l'apparition des symptômes. La prescription initiale médicale doit être réalisée par un neurologue ou un médecin spécialiste exerçant dans un centre de sommeil. Un suivi rapproché en début de traitement par un médecin spécialisé est nécessaire. Lorsque le traitement est équilibré, notre groupe d'experts recommande une consultation annuelle de suivi.

Lorsqu'un traitement apparaît inefficace ou peu efficace, changer de classe thérapeutique peut être utile. Si un traitement n'est que partiellement efficace, afin d'éviter de trop augmenter les posologies, proposer une bithérapie peut être une alternative possible.

\section{Cas particuliers}




\subsection{SJSR et insuffisants rénaux}

A ce jour, aucune recommandation n'existe pour le SJSR chez les patients ayant une insuffisance rénale. Une étude à court terme, chez des patients dialysés, a démontré l'efficacité de la rotigotine à la posologie de 1-3 mg/jour sur la sévérité du SJSR et sur les mouvements périodiques du sommeil et sa bonne tolérance [32].

\subsection{SJSR et femme enceinte}

A ce jour, aucune recommandation n'existe pour le SJSR chez la femme enceinte. Notre groupe d'experts recommande de rechercher et de traiter la carence martiale. Les agonistes dopaminergiques ne sont pas recommandés. Au cas par cas, l'utilisation des opioïdes ou des benzodiazépines peut être discuté avec le gynécologue en prenant en compte le risque de syndrome de sevrage néonatal.

\subsection{SJSR et antidépresseur}

Nous recommandons de revoir avec le psychiatre l'indication du traitement antidépresseur et de traiter l'éventuelle insomnie associée.

\subsection{SJSR et enfants}

Le SJSR de l'enfant doit être pris en charge par un médecin expert du sommeil de l'enfant. Aucune recommandation thérapeutique n'existe à ce jour. La carence martiale doit être systématiquement recherchée et traitée. Les agonistes dopaminergiques ne sont pas recommandés du fait de l'absence de données sur leur efficacité et leur sécurité chez les enfants. Le consensus d'expert retenu est l'utilisation de gabapentine chez l'enfant ayant un SJSR persistant et sévère avec répercussions diurnes importantes (après correction des facteurs aggravants et traitement d'une carence en fer, et après avoir éliminé des comorbidités associées).

\subsection{Les mouvements périodiques du sommeil isolés}

A ce jour, aucune recommandation n'existe pour le traitement des mouvements périodiques du sommeil isolés. Notre consensus d'experts, au vu de nos retours d'expérience, est en faveur d'une abstention thérapeutique pour les mouvements périodiques du sommeil. En cas de mouvements périodiques très sévères en nombre et si ces derniers sont très éveillants, un essai de traitement peut 
être tenté avec un traitement de type anti-épileptique.

\subsection{SJSR et anesthésie}

En cas d'anesthésie, les neuroleptiques, les dérivés sédatifs ou antiémétiques à propriétés antidopaminergiques centrales doivent être évités. Chez un patient ayant un SJSR sévère ou très sévère, il peut être utile en post opératoire de lui proposer un traitement morphinique par patch ou par voie parentérale. En cas de perte sanguine importante, un contrôle du bilan martial est nécessaire. II est recommandé de reprendre le traitement habituel du SJSR dès que possible [33]. 
Tableau 1. Conseils d'hygiène de vie dans le SJSR

- Appliquer des horaires veille-sommeils réguliers

- Se détendre avant l'endormissement

- Eviter les écrans avant l'endormissement

- Ne pas dormir dans des pièces trop chaudes

- Pas d'activité physique le soir

- Eviter l'alcool (notamment vin blanc et champagne), le café, la nicotine

- Avancer l'heure du coucher pour éviter d'être au maximum des symptômes au moment de l'endormissement (quand cela est possible)

- Conseiller une activité intellectuelle minutieuse (par exemple, un horloger de précision qui n'est jamais gêné par son SJSR lorsqu'il est concentré sur son travail) 
Tableau 2. Indication et posologies des traitements du SJSR

\begin{tabular}{|c|c|c|c|c|c|}
\hline \multicolumn{2}{|c|}{ Traitement } & \multicolumn{2}{|c|}{ Posologie (mg) } & \multirow{2}{*}{$\begin{array}{l}\text { AMM dans } \\
\text { l'indication } \\
\text { SJSR }\end{array}$} & \multirow{2}{*}{$\begin{array}{c}\text { Remboursement } \\
\text { dans l'indication } \\
\text { SJSR }\end{array}$} \\
\hline & & $\min$ & $\max$ & & \\
\hline \multirow{3}{*}{$\begin{array}{l}\text { Agoniste } \\
\text { dopaminergique }\end{array}$} & $\begin{array}{l}\text { ADARTREL }^{\circledR} \\
\text { (Ropinirole) }\end{array}$ & 0,25 & 2 & oui & non \\
\hline & $\begin{array}{l}\text { SIFROL }^{\circledR} \\
\text { (Pramipexole) }\end{array}$ & 0,09 & 0,54 & oui & non \\
\hline & $\begin{array}{l}\text { NEUPRO } \\
\text { (Rotigotine) }\end{array}$ & 1 & 3 & oui & non \\
\hline \multirow[t]{2}{*}{ Ligand $\alpha 2 \delta$} & Gabapentine & 100 & $\begin{array}{l}1200 \mathrm{mg} \\
\text { en une } \\
\text { prise }\end{array}$ & non & non \\
\hline & Prégabaline & 25 & 200 & non & non \\
\hline Opioïdes & Tramadol & 50 & 100 & non & non \\
\hline
\end{tabular}




\section{Références}

[1] Cho YW, Allen RP, Earley CJ. Clinical efficacy of ferric carboxymaltose treatment in patients with restless legs syndrome. Sleep Med 2016;25:16-23. doi:10.1016/j.sleep.2016.06.021.

[2] Allen RP, Adler CH, Du W, Butcher A, Bregman DB, Earley CJ. Clinical efficacy and safety of IV ferric carboxymaltose (FCM) treatment of RLS: A multi-centred, placebo-controlled preliminary clinical trial. Sleep Med 2011;12:906-13. doi:10.1016/j.sleep.2011.06.009.

[3] Partinen M, Hirvonen K, Jama L, Alakuijala A, Hublin C, Tamminen I, et al. Efficacy and safety of pramipexole in idiopathic restless legs syndrome: A polysomnographic dose-finding studyThe PRELUDE study. Sleep Med 2006;7:407-17. doi:10.1016/j.sleep.2006.03.011.

[4] Ferini-Strambi L, Aarskog D, Partinen M, Chaudhuri KR, Sohr M, Verri D, et al. Effect of pramipexole on RLS symptoms and sleep: A randomized, double-blind, placebo-controlled trial. Sleep Med 2008;9:874-81. doi:10.1016/j.sleep.2008.09.001.

[5] Trenkwalder C, Stiasny-Kolster K, Kupsch A, Oertel WH, Koester J, Reess J, et al. Controlled withdrawal of pramipexole after 6 months of open-label treatment in patients with restless legs syndrome. Mov Disord 2006;21:1404-10. doi:10.1002/mds.20983.

[6] Montagna P, Hornyak M, Ulfberg J, Hong SB, Koester J, Crespi G, et al. Randomized trial of pramipexole for patients with restless legs syndrome (RLS) and RLS-related impairment of mood. Sleep Med 2011;12:34-40. doi:10.1016/j.sleep.2010.08.005.

[7] Högl B, Garcia-Borreguero D, Trenkwalder C, Ferini-Strambi L, Hening W, Poewe W, et al. Efficacy and augmentation during 6 months of double-blind pramipexole for restless legs syndrome. Sleep Med 2011;12:351-60. doi:10.1016/j.sleep.2010.12.007.

[8] Aurora RN, Kristo DA, Bista SR, Rowley JA, Zak RS, Casey KR, et al. The Treatment of Restless Legs Syndrome and Periodic Limb Movement Disorder in Adults_An Update for 2012: Practice Parameters with an Evidence-Based Systematic Review and Meta-Analyses. SLEEP 2012. doi:10.5665/sleep.1988.

[9] Garcia-Borreguero D, Ferini-Strambi L, Kohnen R, O'Keeffe S, Trenkwalder C, Högl B, et al. European guidelines on management of restless legs syndrome: report of a joint task force by the European Federation of Neurological Societies, the European Neurological Society and the European Sleep Research Society. Eur J Neurol 2012;19:1385-96. doi:10.1111/j.14681331.2012.03853.x.

[10] Oertel WH, Benes H, Garcia-Borreguero D, Geisler P, Högl B, Saletu B, et al. Efficacy of rotigotine transdermal system in severe restless legs syndrome: A randomized, double-blind, placebo-controlled, six-week dose-finding trial in Europe. Sleep Med 2008;9:228-39. doi:10.1016/j.sleep.2007.04.010.

[11] Trenkwalder C, Beneš H, Poewe W, Oertel WH, Garcia-Borreguero D, de Weerd AW, et al. Efficacy of rotigotine for treatment of moderate-to-severe restless legs syndrome: a randomised, double-blind, placebo-controlled trial. Lancet Neurol 2008;7:595-604. doi:10.1016/S14744422(08)70112-1.

[12] Oertel WH, Beneš H, Garcia-Borreguero D, Högl B, Poewe W, Montagna P, et al. Rotigotine transdermal patch in moderate to severe idiopathic restless legs syndrome: A randomized, 
placebo-controlled polysomnographic study. Sleep Med 2010;11:848-56.

doi:10.1016/j.sleep.2010.02.014.

[13] Oertel WH, Benes H, Garcia-Borreguero D, Geisler P, Högl B, Trenkwalder C, et al. One year open-label safety and efficacy trial with rotigotine transdermal patch in moderate to severe idiopathic restless legs syndrome. Sleep Med 2008;9:865-73. doi:10.1016/j.sleep.2008.04.012.

[14] Oertel W, Trenkwalder C, Beneš H, Ferini-Strambi L, Högl B, Poewe W, et al. Long-term safety and efficacy of rotigotine transdermal patch for moderate-to-severe idiopathic restless legs syndrome: a 5-year open-label extension study. Lancet Neurol 2011;10:710-20. doi:10.1016/S1474-4422(11)70127-2.

[15] Cornelius JR, Tippmann-Peikert M, Slocumb NL, Frerichs CF, Silber MH. Impulse control disorders with the use of dopaminergic agents in restless legs syndrome: a case-control study. Sleep 2010;33:81-7.

[16] Ondo WG, Lai D. Predictors of impulsivity and reward seeking behavior with dopamine agonists. Parkinsonism Relat Disord 2008;14:28-32. doi:10.1016/j.parkreldis.2007.05.006.

[17] Driver-Dunckley ED, Noble BN, Hentz JG, Evidente VGH, Caviness JN, Parish J, et al. Gambling and Increased Sexual Desire With Dopaminergic Medications in Restless Legs Syndrome: Clin Neuropharmacol 2007;30:249-55. doi:10.1097/wnf.0b013e31804c780e.

[18] O'Sullivan SS, Evans AH, Lees AJ. Dopamine Dysregulation Syndrome: An Overview of its Epidemiology, Mechanisms and Management. CNS Drugs 2009;23:157-70. doi:10.2165/00023210-200923020-00005.

[19] Hornyak M, Trenkwalder C, Kohnen R, Scholz H. Efficacy and safety of dopamine agonists in restless legs syndrome. Sleep Med 2012;13:228-36. doi:10.1016/j.sleep.2011.09.013.

[20] Garcia-Borreguero D, Larrosa O, de la Llave Y, Verger K, Masramon X, Hernandez G. Treatment of restless legs syndrome with gabapentin: a double-blind, cross-over study. Neurology 2002;59:1573-9.

[21] Happe S, Sauter C, Klösch G, Saletu B, Zeitlhofer J. Gabapentin versus Ropinirole in the Treatment of Idiopathic Restless Legs Syndrome. Neuropsychobiology 2003;48:82-6. doi:10.1159/000072882.

[22] Inoue Y, Hirata K, Uchimura N, Kuroda K, Hattori N, Takeuchi M. Gabapentin enacarbil in Japanese patients with restless legs syndrome: a 12-week, randomized, double-blind, placebocontrolled, parallel-group study. Curr Med Res Opin 2013;29:13-21. doi:10.1185/03007995.2012.746217.

[23] Bogan RK, Lee DO, Buchfuhrer MJ, Jaros MJ, Kim R, Shang G. Treatment response to sleep, pain, and mood disturbance and their correlation with sleep disturbance in adult patients with moderate-to-severe primary restless legs syndrome: Pooled analyses from 3 trials of gabapentin enacarbil. Ann Med 2015;47:269-77. doi:10.3109/07853890.2015.1025825.

[24] Garcia-Borreguero D, Silber MH, Winkelman JW, Högl B, Bainbridge J, Buchfuhrer M, et al. Guidelines for the first-line treatment of restless legs syndrome/Willis-Ekbom disease, prevention and treatment of dopaminergic augmentation: a combined task force of the IRLSSG, 
EURLSSG, and the RLS-foundation. Sleep Med 2016;21:1-11.

doi:10.1016/j.sleep.2016.01.017.

[25] Allen R, Chen C, Soaita A, Wohlberg C, Knapp L, Peterson BT, et al. A randomized, doubleblind, 6-week, dose-ranging study of pregabalin in patients with restless legs syndrome. Sleep Med 2010;11:512-9. doi:10.1016/j.sleep.2010.03.003.

[26] Garcia-Borreguero D, Larrosa O, Williams A-M, Albares J, Pascual M, Palacios JC, et al. Treatment of restless legs syndrome with pregabalin: A double-blind, placebo-controlled study. Neurology 2010;74:1897-904. doi:10.1212/WNL.0b013e3181e1ce73.

[27] Garcia-Borreguero D, Patrick J, DuBrava S, Becker PM, Lankford A, Chen C, et al. Pregabalin Versus Pramipexole: Effects on Sleep Disturbance in Restless Legs Syndrome. SLEEP 2014. doi:10.5665/sleep.3558.

[28] Trenkwalder C, Beneš H, Grote L, García-Borreguero D, Högl B, Hopp M, et al. Prolonged release oxycodone-naloxone for treatment of severe restless legs syndrome after failure of previous treatment: a double-blind, randomised, placebo-controlled trial with an open-label extension. Lancet Neurol 2013;12:1141-50. doi:10.1016/S1474-4422(13)70239-4.

[29] Walters AS, Wagner ML, Hening WA, Grasing K, Mills R, Chokroverty S, et al. Successful treatment of the idiopathic restless legs syndrome in a randomized double-blind trial of oxycodone versus placebo. Sleep 1993;16:327-32.

[30] Allen RP, Chen C, Garcia-Borreguero D, Polo O, DuBrava S, Miceli J, et al. Comparison of Pregabalin with Pramipexole for Restless Legs Syndrome. N Engl J Med 2014;370:621-31. doi:10.1056/NEJMoa1303646.

[31] Allen RP, Chen C, Garcia-Borreguero D, Polo O, DuBrava S, Miceli J, et al. Comparison of Pregabalin with Pramipexole for Restless Legs Syndrome. N Engl J Med 2014;370:621-31. doi:10.1056/NEJMoa1303646.

[32] Dauvilliers $Y$, Benes H, Partinen M, Rauta V, Rifkin D, Dohin E, et al. Rotigotine in Hemodialysis-Associated Restless Legs Syndrome: A Randomized Controlled Trial. Am J Kidney Dis 2016;68:434-43. doi:10.1053/j.ajkd.2015.12.027.

[33] Karroum EG, Raux M, Riou B, Arnulf I. Implications anesthésiques du syndrome des jambes sans repos $\square$ : observations cliniques et recommandations pratiques. Ann Fr Anesth Réanimation 2010;29:920-4. doi:10.1016/j.annfar.2010.09.002. 


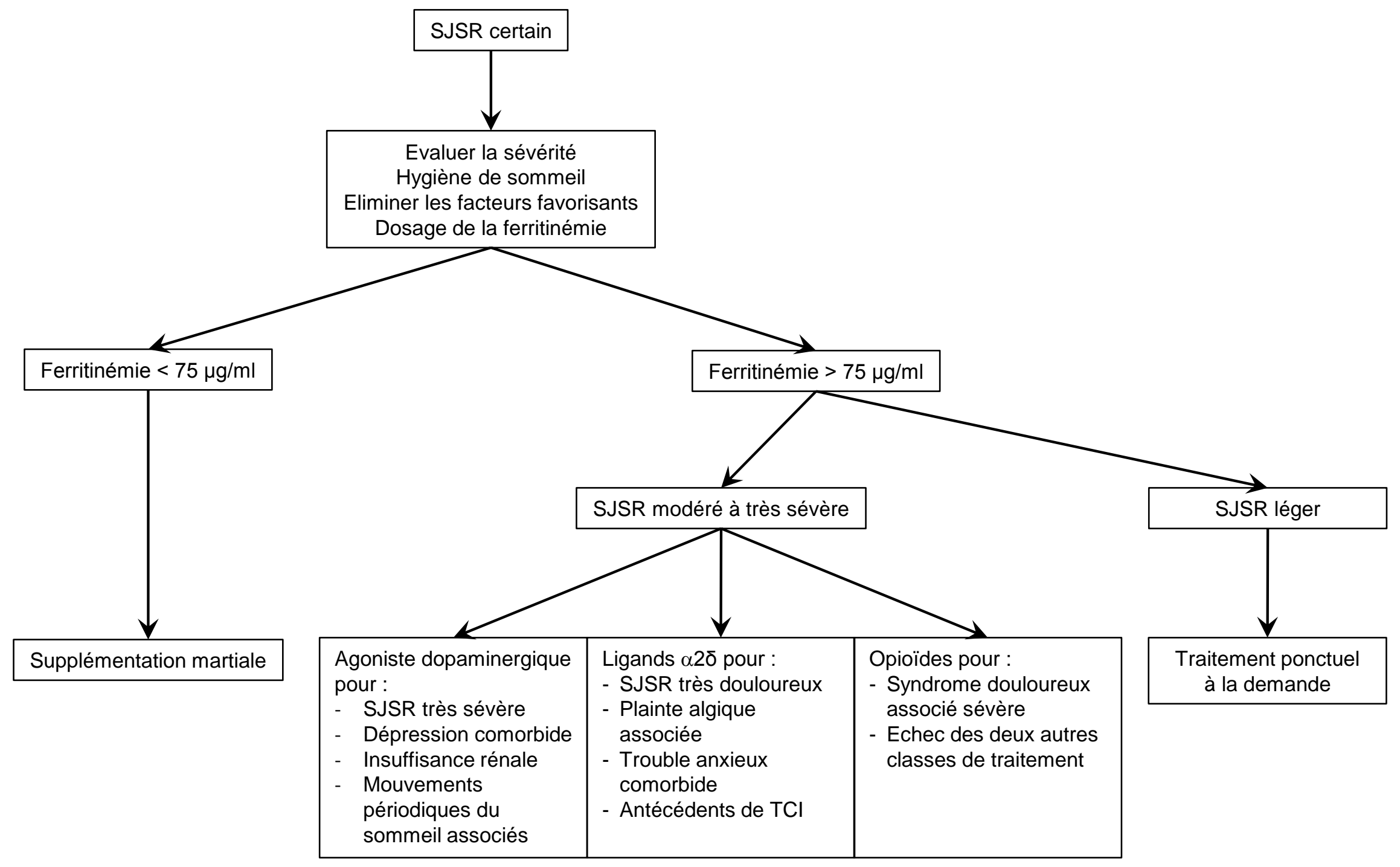

Figure 1. Arbre thérapeutique 